\title{
Prompt neutron emission and energy balance in ${ }^{235} \mathrm{U}(\mathrm{n}, \mathrm{f})$
}

\author{
Alf Göök ${ }^{\mathrm{a}}$, Franz-Josef Hambsch, and Stephan Oberstedt \\ European Commision, Joint Research Centre, Directorate G - Nuclear Safety and Security, Unit G.2, Retieseweg 111, \\ 2440 Geel, Belgium
}

\begin{abstract}
Investigations of prompt fission neutron (PFN) emission are of importance in understanding the fission process in general and the sharing of excitation energy among the fission fragments in particular. Experimental activities at JRC-Geel on PFN emission in response to OECD/NEA nuclear data requests is presented in this contribution. The focus lies on on-going investigations of PFN emission from the reaction ${ }^{235} \mathrm{U}(\mathrm{n}, \mathrm{f})$ in the region of the resolved resonances taking place at the GELINA facility. For this reaction strong fluctuations of fission fragment mass distributions and mean total kinetic energy have been observed as a function of incident neutron energy in the resonance region. In addition, fluctuations of prompt neutron multiplicities have also been observed. The goal of the present study is to verify the current knowledge of PFN multiplicity fluctuations and to study correlations with fission fragment properties. The experiment employs a scintillation detector array for neutron detection, while fission fragment properties are determined via the double kinetic energy technique using a position sensitive twin ionization chamber.

Results on PFN multiplicity correlations with fission fragment properties from the present study show significant differences compared to earlier studies on this reaction, induced by thermal neutrons. Specifically, the total kinetic energy dependence of the neutron multiplicity per fission shows an inverse slope $\partial \mathrm{TKE} / \partial \bar{v}$ approximately $35 \%$ weaker than observed in earlier studies of thermal neutron induced fission on ${ }^{235} \mathrm{U}$. The inverse slope is related to the energy carried away per emitted neutron and is, thereby, closely connected to the energy balance of the fission reaction. The present result should have strong impact on the modeling of both prompt neutron and prompt $\gamma$-ray emission in fission of the ${ }^{236} \mathrm{U}$ compound nucleus.
\end{abstract}

\section{Introduction}

Experimental investigations of prompt fission neutrons and fission fragment properties in resonance neutron induced fission on ${ }^{235} \mathrm{U}$ are taking place at the GELINA facility of the JRC-Geel. Improved knowledge of the properties of prompt fission neutrons, their multiplicities, as well as their energy and angular distributions could give answers to questions related not only to the neutron emission itself, but also to questions relevant to the formation of the fission fragments, the sharing of excitation energy among them and the time scale of the process. For nuclear modeling and improved evaluation of nuclear data the knowledge about fluctuations in the prompt neutron multiplicity as a function of incident neutron energy is requested for the major actinides ${ }^{235} \mathrm{U}$ and ${ }^{239} \mathrm{Pu}[1,2]$. Fluctuations in fission fragment mass and total kinetic energy (TKE) in both isotopes have been observed in resonance neutron induced fission $[3,4]$. Independently, fluctuations in the number of emitted neutrons have also been observed [5]. In view of the fact that both the neutron multiplicity and fission fragment properties have been found to vary it is necessary to study the correlations of prompt neutron multiplicity and fission fragments properties in the resonance region.

Efforts to calculate PFN and fragment correlations using Monte-Carlo technique have been quite successful in reproducing available experimental data [6-8]. However,

a e-mail: alf .gook@ec. europa.eu in the case of ${ }^{235} \mathrm{U}(\mathrm{n}, \mathrm{f})$ difficulties have been encountered [8], especially regarding the dependency of $\bar{v}$ on the fragment TKE. In Ref. [9] possible deficiencies in the experimental data where pointed out. This makes it interesting to revisit PFN and fragment correlations in ${ }^{235} \mathrm{U}(\mathrm{n}, \mathrm{f})$ experimentally.

\section{Experimental setup}

The experimental setup is schematically illustrated in Fig. 1. It consists of two parts, a position sensitive twin ionization chamber for fission fragment detection and an array of scintillation neutron detectors (SCINTIA). A detailed description of the position sensitive ionization chamber can be found in Ref. [10]. The ${ }^{235} \mathrm{U}$ target placed inside the ionization chamber is a circular $7 \mathrm{~cm}$ diameter spot of $67.2 \mu \mathrm{gU} / \mathrm{cm}^{2} \mathrm{UF}_{4}$ evaporated onto a $27 \mu \mathrm{g} / \mathrm{cm}^{2}$ polyimide $+50 \mu \mathrm{g} / \mathrm{cm}^{2}$ gold backing. The chamber is placed so that the incident neutrons travel $\sim 9.2 \mathrm{~m}$ from the GELINA target before reaching the ${ }^{235} \mathrm{U}$ target, which allows the incident neutron energy to be determined by the time-of-flight (ToF) technique. A dedicated campaign, with GELINA operating at $50 \mathrm{~Hz}$ repetition frequency gave access to the thermal component of the incident neutron spectrum in ToF. Data from this run was used for calibrations of the fission fragment detector to well known thermal fission fragment characteristics. The detectors of SCINTIA are of three different types but they all have similar characteristics in terms of detection efficiency, pulse shape discrimination properties 


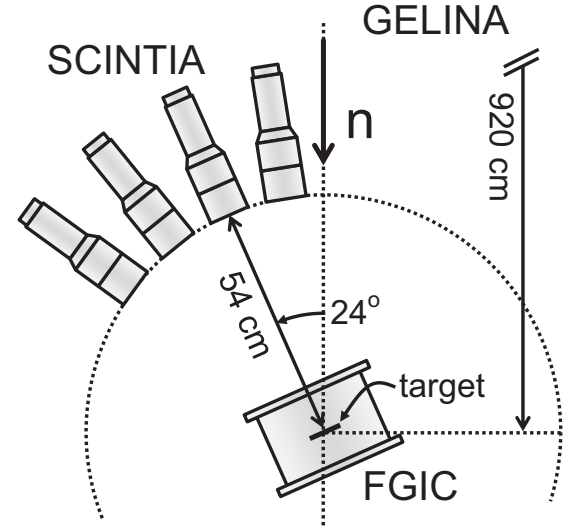

Figure 1. Schematic view of the experimental setup.

Table 1. Summary of the neutron detector array SCINTIA. Three different type of detectors are used; the EJ-301 is an NE-213 equivalent liquid scintillator, while the p-therphenyl and the stilbene are organic crystal scintillators. The detector orientation axis is given by unit vectors in the coordinate system of the ionization chamber, with the z-axis perpendicular to the target plane.

\begin{tabular}{clcc}
\hline Nr. & Type & Size & Orientation $(\boldsymbol{x}, \boldsymbol{y}, \boldsymbol{z})$ \\
\hline 0 & p-therphenyl & $3^{\prime \prime}$ & $(-0.399,0.000,0.917)$ \\
1 & EJ-301 & $4^{\prime \prime}$ & $(-0.326,0.577,0.749)$ \\
2 & EJ-301 & $4^{\prime \prime}$ & $(-0.382,0.289,0.878)$ \\
3 & EJ-301 & $5^{\prime \prime}$ & $(-0.654,0.327,0.682)$ \\
4 & EJ-301 & $5^{\prime \prime}$ & $(-0.055,0.326,0.944)$ \\
5 & stilbene & $3^{\prime \prime}$ & $(0.137,0.000,0.991)$ \\
6 & p-therphenyl & $3^{\prime \prime}$ & $(-0.107,0.000,0.994)$ \\
7 & p-therphenyl & $3^{\prime \prime}$ & $(-0.667,0.000,0.745)$ \\
8 & p-therphenyl & $3^{\prime \prime}$ & $(0.000,0.000,-1.000)$ \\
9 & EJ-301 & $4^{\prime \prime}$ & $(-0.373,-0.353,0.858)$ \\
10 & EJ-301 & $5^{\prime \prime}$ & $(-0.048,-0.332,0.942)$ \\
11 & EJ-301 & $5^{\prime \prime}$ & $(-0.661,-0.339,0.670)$ \\
\hline
\end{tabular}

and timing resolution. Detector type and orientation relative to the chamber is summarized in Table 1.

\section{Data analysis}

\subsection{Analysis of PFN data}

Events in the scintialltion detectors corresponding to neutrons are selected by means of pulse-shape discrimination (PSD). The PFN energy is measured by means of the ToF technique, with a resolution of $\sim 1 \mathrm{~ns}$ (FWHM). The fission process is in addition to PFNs also accompanied by prompt fission $\gamma$-rays, which might be detected in the scintialltion detector. Most of the $\gamma$-emission takes place within a few ns after the instant of fission. Therefore, the range of high-energy neutrons is most sensitive to false events induced by $\gamma$-rays. The PSD is not able to give a clean discrimination between $\gamma$-rays and neutron events for pulse heights corresponding to a proton recoil energy smaller than $\sim 1 \mathrm{MeV}$. In order to reduce the background caused by the $\gamma$-emission a dynamic light threshold [11] is applied. Based on experimentally determined relation $L\left(E_{p}\right)$ between scintillation-light output $L$ and recoil proton energy $E_{p}$ the lower threshold for a given neutron energy $E_{n}$ is determined by choosing a critical recoil

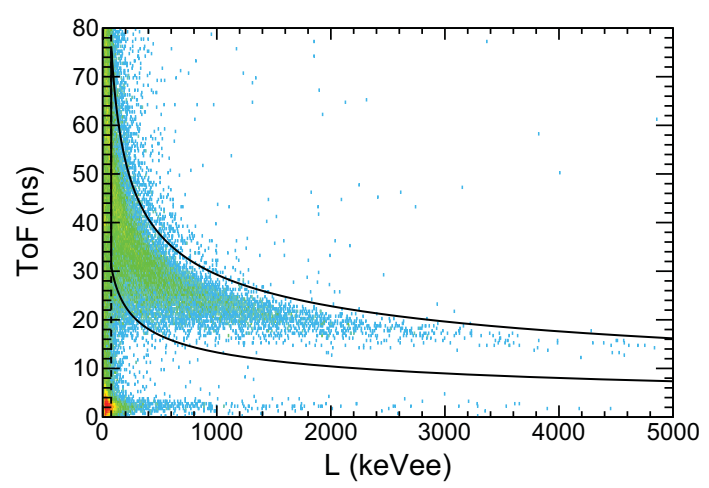

Figure 2. PFN time-of-flight vs. light output in one of the neutron detectors. The dynamic light output selection is shown by the full black line, the vertical dashed black line corresponds to the $0.5 \mathrm{MeV}$ proton recoil energy pulse-height threshold.

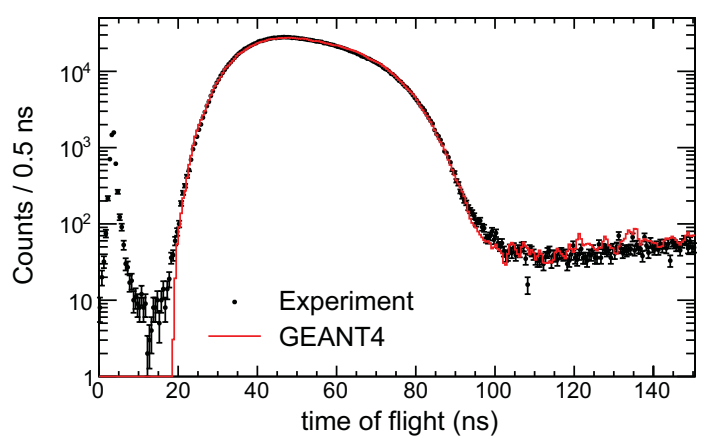

Figure 3. Experimental and simulated ToF spectrum of ${ }^{252} \mathrm{Cf}(\mathrm{sf})$ PFNs in the detector calibration setup.

angle $\theta_{R}:=60^{\circ}$

$$
L_{\min }\left(E_{n}\right)=L\left(E_{p}\left(\theta_{R}\right)\right), \quad E_{p}\left(\theta_{R}\right)=E_{n} \cos ^{2} \theta_{R} .
$$

In addition, a pulse-height threshold corresponding to $0.5 \mathrm{MeV}$ proton recoil energy is applied to each detector. The upper threshold is set at

$$
L_{\max }\left(E_{n}\right)=L\left(E_{p}\right)+3 \cdot \sigma_{L}\left(E_{p}\right), \quad E_{p}=E_{n}
$$

where $\sigma_{L}\left(E_{p}\right)$ is the experimentally determined light output resolution of the detector. The selection is exemplified in Fig. 2.

Each detector in the SCINTIA array has been characterized using the PFNs from ${ }^{252} \mathrm{Cf}(\mathrm{sf})$, with experimental setup and procedures described in Ref. [12]. To correct for the energy dependent detection efficiency of the detectors and multiple scattering of neutrons on the surrounding materials the setup is modeled in GEANT4. The simulation uses the experimentally determined proton light output functions, for other recoil-particle species literature data is used $[13,14]$. The Monte-Carlo model has been validated against the ${ }^{252} \mathrm{Cf}(\mathrm{sf}) \mathrm{PFNS}$ [15]. In Fig. 3 the simulated time-of-flight spectrum from the detector calibration setup is compared to the experimental data. The simulated spectrum agrees with the experimental one within $\pm 4 \%$ for neutron energies below $10 \mathrm{MeV}$. Correction curves were calculated with the GEANT4 code as the ratio of the observed neutron spectrum in each detector to the input spectrum emitted from the source position in the experimental setup. The input spectrum used for the calculation was taken from the recent evaluation in Ref. [16]. 


\subsection{Analysis of fission fragment data}

The direct experimental fission fragment observables are the energies deposited as ionization of the chambers counting gas and the orientation of the fission axis in space. The energy deposited in the counting gas is related to the energies of the fission fragment after prompt neutron emission by correcting for angular dependent energy loss in the target and backing foil and pulse height defect in the counting gas. The energy scale was calibrated to the recommended literature value $\overline{\mathrm{TKE}}=170.5 \mathrm{MeV}$ [17] using the measured thermal neutron induced fission data. The fission fragment mass and kinetic energy before neutron emission is obtained by means of the well established double kinetic energy (2E) technique, which relates the masses $m_{1,2}^{*}$ and energies $E_{1,2}^{*}$ before neutron emission in a binary fission event

$$
m_{1,2}^{*}=m_{c n} \cdot \frac{E_{2,1}^{*}}{E_{1}^{*}+E_{2}^{*}},
$$

where $m_{c n}=236$ is the mass number of the compound nucleus undergoing fission. Under the assumption of isotropic emission from fully accelerated fragments, the energies before $E^{*}$ neutron emission are related to the energies $E$ after neutron emission according to

$$
E^{*}=E \cdot \frac{m^{*}}{m^{*}-\bar{v}\left(m^{*}, \mathrm{TKE}\right)},
$$

where $\bar{v}$ is the number of neutrons emitted by the fragment. The dependence of $\bar{v}$ on mass and TKE can only be derived from the data once the 2E-analysis is completed. As initial assumption we have used the evaluated data on $\bar{v}\left(m^{*}\right)$ from Wahl [18] and the parameterization

$$
\begin{aligned}
\bar{v}\left(m^{*}, \mathrm{TKE}\right) & =\bar{v}\left(m^{*}\right)+\frac{\bar{v}\left(m^{*}\right)}{\overline{\bar{v}}\left(m^{*}\right)+\bar{v}\left(m_{c n}-m^{*}\right)} \cdot \Delta_{\mathrm{TKE}}, \\
\Delta_{\mathrm{TKE}} & =\frac{\overline{\operatorname{TKE}}\left(m^{*}\right)-\mathrm{TKE}}{E_{\mathrm{sep}}},
\end{aligned}
$$

where $E_{\text {sep }}=8.6 \mathrm{MeV} / \mathrm{n}$ is the average energy necessary to emit a neutron [19]. The analysis was later repeated using the results on $\bar{v}\left(m^{*}\right)$ and $E_{\text {sep }}=8.51 \mathrm{MeV} / \mathrm{n}$ derived from the data. No significant changes in the results were observed between the two analyses. In the 2E-technique the main contribution to the mass resolution is the correction for the neutron evaporation. In this work the mass resolution is 5-6 u (FWHM), determined by comparing the measured thermal mass yield to data from Ref. [20].

\subsection{Analysis of fission fragment and neutron coincident data}

To arrive at average neutron multiplicities per fragment the coincident data was treated according to the procedure outlined in our work on ${ }^{252} \mathrm{Cf}(\mathrm{sf})$ [21]. The measured properties of the prompt neutrons are transformed into the rest frame of the fully accelerated fragment. A selection of events where the neutron is emitted with an angle $\vartheta_{c . m .} \leq 90^{\circ}$ in the fission fragment rest-frame is made. Corrections for the recoil energy imparted to the fission fragment by the detected neutron [22] and detection of neutrons originating from the complementary fragment are made.
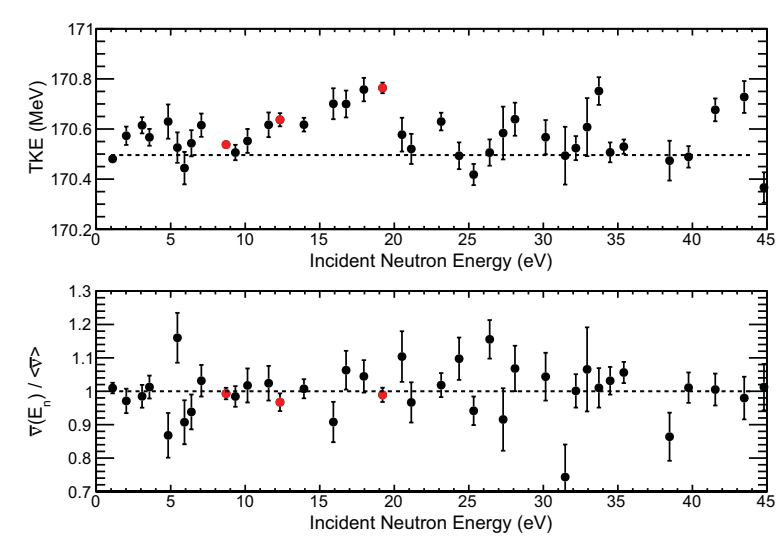

Figure 4. Average TKE as a function of incident neutron energy for given resonance or resonance group in the range up to $45 \mathrm{eV}$ (top), the dashed line refers to the thermal value. Neutron multiplicity for the same resonances or resonance groups relative to the weighted average neutron multiplicity (bottom). The red points highlight strong resonances in the fission cross section of ${ }^{235} \mathrm{U}$.

\section{Experimental results \& discussion}

The data presented in this contribution stems from the first campaign of the experiment and represent $\sim 25 \%$ of the expected final number of events. The second campaign finished recently and the analysis of data from this campaign is still not in it's final stage. A third campaign is planned for the second half of 2016.

\subsection{Fission fragment properties and neutron multiplicity in the resolved resonance region}

The top panel of Fig. 4 shows the average TKE as a function of incident neutron energy in the range up to $45 \mathrm{eV}$. Each point corresponds to either a single resonance or resonance group. The red points highlight the strongest resonances in the fission cross section of ${ }^{235} \mathrm{U}$. A clear fluctuating behavior exceeding the experimental uncertainty is observed, which confirms the findings in Ref. [3]. However, the statistical significance is improved in the present work leading to a clearer picture of the energy dependent changes compared to the earlier work. The lower panel of Fig. 4 shows the neutron multiplicity for a given resonance or resonance group relative to the weighted average neutron multiplicity. Again, the strongest resonances are highlighted in red. At the present stage of data collection no statistically significant fluctuation of the neutron multiplicity from resonance to resonance is observed.

The fluctuating behavior of the TKE can be understood when examining the fission fragment mass distributions. In Fig. 5 the difference in mass yield compared to thermal neutron induced fission is shown for a gate around the resonances at $19.3 \mathrm{eV}$, the same difference is shown also for the sum of data in between resonances. The $19.3 \mathrm{eV}$ resonance's mass distribution shows a sizable increase in yield in the region $A_{H} \in(125,135) \mathrm{u}$. In this mass region $\operatorname{TKE}(A)$ is at its maximum, which explains the apparent increase in TKE for this resonance when integrating over the mass distribution. Contrary to the strongly mass dependent $\overline{\operatorname{TKE}}(A)$, the average neutron multiplicity per fission is only weakly dependent on mass split. However, in the mass region $A_{H} \in(125,135) \mathrm{u}$ the average neutron 

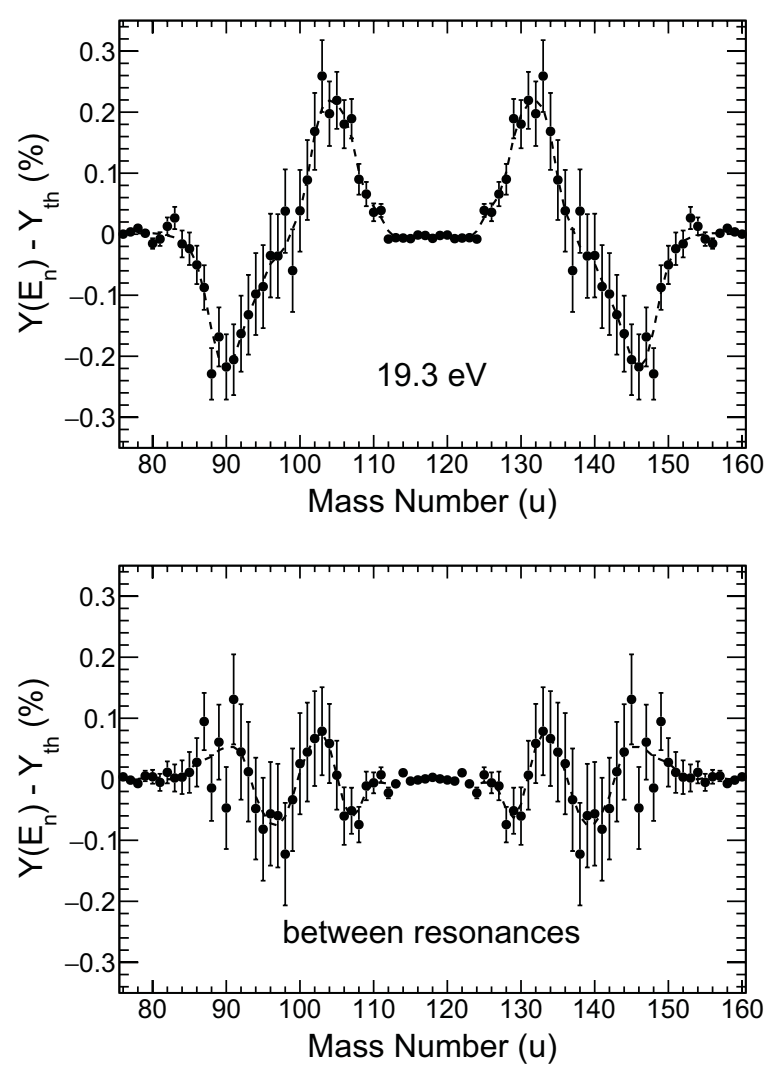

Figure 5. Difference in mass yield to the thermal neutron induced fission of the $19.3 \mathrm{eV}$ resonance (top) as well as for selection of data between the resonances (bottom). The dashed lines shows the data smoothed over two mass units, drawn to guide the eye.

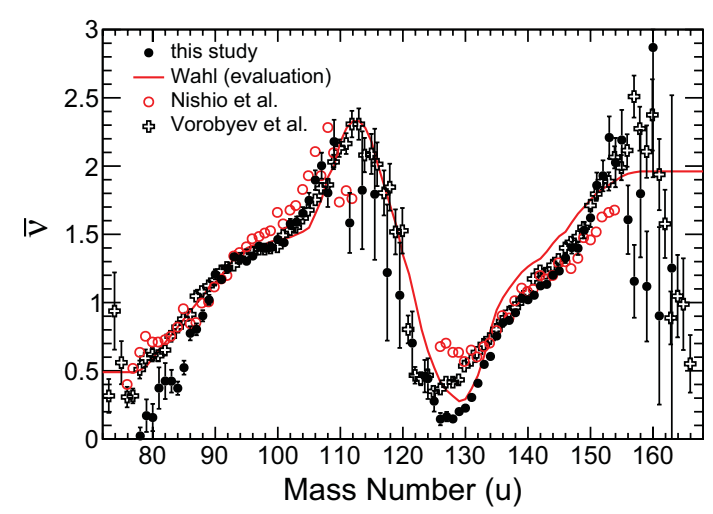

Figure 6. The average neutron multiplicity per fragment as a function of the fragment mass from this study compared to data from Refs. [23,24] and the evaluation by Wahl [18].

multiplicity per fission shows a pronounced minimum (cf. Sect. 4.2). Hence, the increased yield in this mass region should have an impact also on the total neutron multiplicity per fission for certain resonances.

\subsection{Correlations between neutron multiplicity and fission fragment properties}

The results presented in this section represent data summed over fissions induced by neutrons in the energy range between $0.3 \mathrm{eV}$ and $60 \mathrm{keV}$, the mean neutron energy that induce fission is $1.6 \mathrm{keV}$.

The average neutron multiplicity as a function of the fragment mass is shown in Fig. 6. For comparison

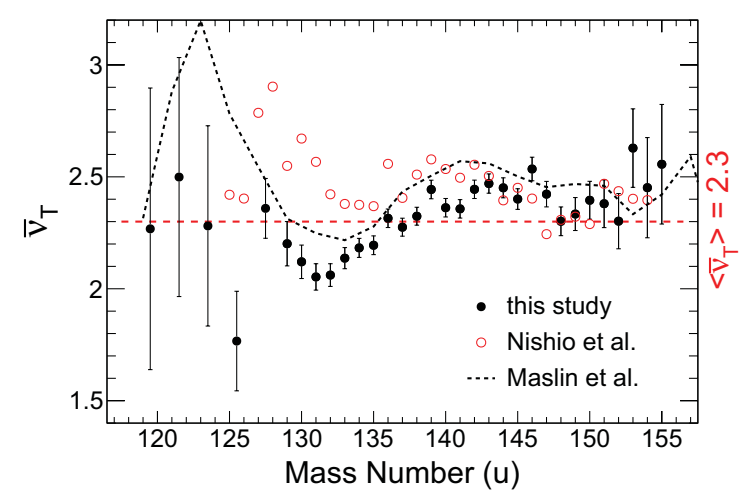

Figure 7. The average neutron multiplicity per fission as a function of the heavy fragment mass from this study compared to data from Refs. [23,25]. The dashed red line indicates the mean multiplicity per fission extracted from the data.

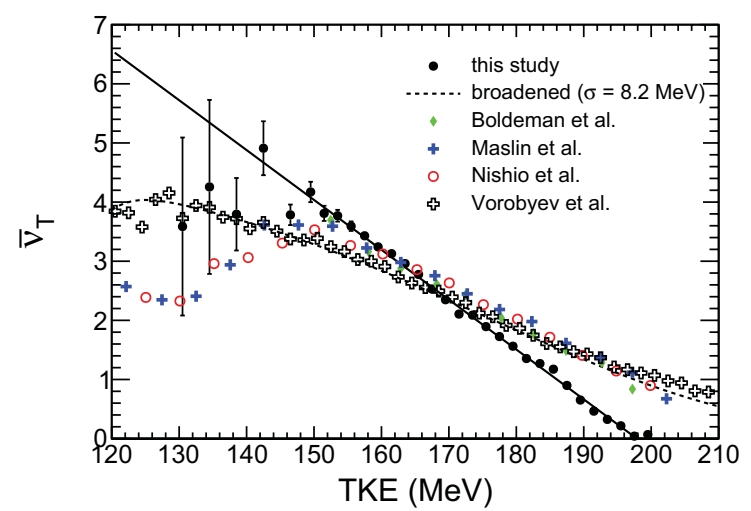

Figure 8. Dependence of prompt neutron multiplicity per fission on the fragment TKE obtained in this study, compared to data from Refs. [23-26]. The data of Ref. [24] has been re-binned for better clarity of the figure. The full black line is a linear least squared fit to the data from this study, which corresponds to an inverse slope of $-\partial \mathrm{TKE} / \partial \bar{v}=11.86 \mathrm{MeV} / \mathrm{n}$. The dotted black line is the result obtained by folding the present data set with a gaussian TKE resolution function with $\sigma=8.2 \mathrm{MeV}$.

experimental data from Refs. [23,24] and the evaluation from Ref. [18] is also show in the figure. The general shape is reproduced in this work, however the minima around mass number $\sim 80 \mathrm{u}$ for the light fragments and $\sim 130 \mathrm{u}$ for the heavy fragments appear more pronounced in the present data. The average neutron multiplicity per fission is shown as a function of the heavy fragment mass in Fig. 7. A pronounced minimum close to heavy fragment mass $A_{H} \sim 132 \mathrm{u}$ observed. The shape of the present data agrees very well with the data from Maslin et al. [25], although the absolute values are $~ 5 \%$ lower.

In Fig. 8 the average neutron multiplicity per fission is plotted as a function of the fragment TKE. As expected, a close to linear dependence is observed, except for at low TKE. A least square fit, indicated by the full black line in Fig. 8, results in $-\partial \mathrm{TKE} / \partial \bar{v}=11.86 \mathrm{MeV} / \mathrm{n}$. This value is substantially lower than the values 16.7-18.5 MeV/n determined in earlier studies, performed at thermal incident neutron energies [23-26]. This inverse slope is closely related to the energy cost to emit a neutron (although it should not be directly interpreted as this quantity [19]). It is not expected that the small increase in the excitation energy of the compound nucleus 


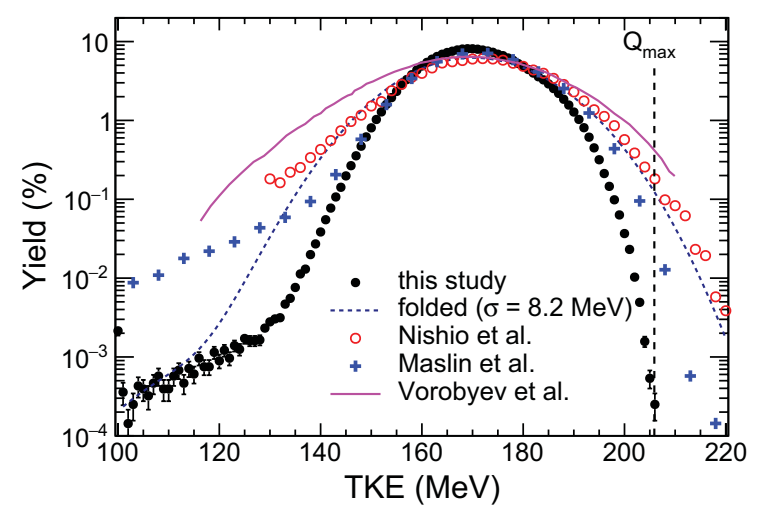

Figure 9. Distribution of the fragment TKE obtained in this study compared to data of Refs. [23-25]. The dotted black line is the result obtained by folding the present data set with a gaussian resolution function with $\sigma=8.2 \mathrm{MeV}$, necessary to reproduce the data of Nishio et al. The vertical dashed line labeled $\mathrm{Q}_{\max }$ indicates the maximum available energy as determined by the fragmentation where the heavy fragment is the doubly magic ${ }^{132}$ Sn nucleus [27].

in this experiment would have an influence on the energy cost to emit a neutron. Hence, we propose a different interpretation, which is purely of instrumental nature. In Fig. 9 the fission fragment TKE distribution from the present measurement is compared to the data of Refs. [23-25]. The distribution from Refs. [23-25] appear broadened. Substantial amounts of yields are found above the maximum available energy, as determined by the Q-value for the fragmentation where the heavy fragment is the doubly magic ${ }^{132} \mathrm{Sn}$ nucleus. Since the TKE cannot physically exceed the maximum available energy the TKE distributions from Refs. [23-25] must suffer from substantial resolution broadening. The dashed line in Fig. 9 represents the result of folding the data from this study with a gausian resolution function in order to reproduce the distribution of Nishio et al. If the same broadening is applied when determining $\bar{v}$ (TKE) the dotted line in Fig. 8 is obtained. This reproduces qualitatively features in the literature data, i.e., a less steep slope and $\bar{v}$ different from zero at the maximum available energy. There are discrepancies at low TKE where the data of Refs. [23,25] show a strong decrease in $\bar{\nu}$, that cannot be explained by the resolution broadening. However, examining Fig. 9 tailings of the TKE distributions can be observed. Events belonging to this tailing are likely due to scattering of the fission fragments in the target foil and/or surrounding materials, as already noted by Maslin et al [25]. The neutron emission from such energy degraded fission events is expected to be close to the average value. This is consistent with the observed decrease in $\bar{v}$ at lower TKE as the tailing become more and more dominant in the yield. The tailing is present also in the data from this study, although at much lower intensities compared to the data of Refs. [23,25]. Due to the presence of the tailing we cannot conclude that the decrease in slope of $\bar{v}$ below $\mathrm{TKE} \sim 140 \mathrm{MeV}$ is of a physical origin.

In order to relate the slope of $\bar{v}$ as a function of the TKE to the energy cost per neutron it must be studied for individual mass splits. A close to linear dependence of $\bar{v}$ on TKE was found for all mass splits where data is available. In Fig. 10 the result of least square fits to

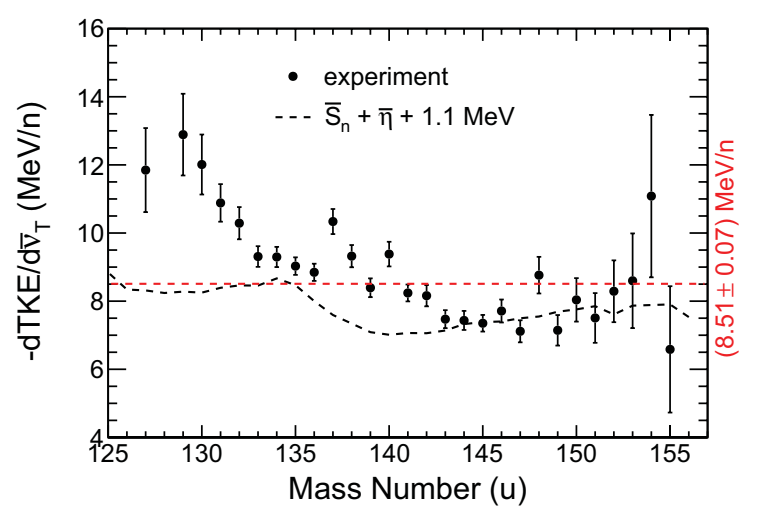

Figure 10. Inverse slope of the neutron multiplicity per fission $\partial \mathrm{TKE} / \partial \bar{v}_{T}$ or the energy cost per emitted neutron as a function of the heavy fragment mass. The dashed red line indicates the weighted average $(8.51 \pm 0.07) \mathrm{MeV} / \mathrm{n}$ of the experimental points. The dashed black line is a calculation based on formulas from Ref. [19] using tabulated neutron binding energies [27] and mean neutron kinetic energies from the present experiment.

the data is presented, the inverse of the slope is plotted as a function of the heavy fragment mass number of the mass split. The results shows a rather flat behaviour above $A_{H} \sim 133 \mathrm{u}$, with an apparent increase when moving towards symmetric mass split. The weighted average value $(8.51 \pm 0.07) \mathrm{MeV} / \mathrm{n}$ is the average energy necessary to emit a neutron and is in close agreement with the initial value $8.6 \mathrm{MeV} / \mathrm{n}$ assumed in the analysis. Based on energy conservation the energy necessary to emit a neutron may also be calculated [19] using tabulated neutron binding energies [27] and experimental values of the mean neutron energy in the fragment rest frame. The result of such a calculation is also presented in Fig. 10. The agreement is fair, although the calculation underestimates the data points, specifically towards symmetric mass splits. The calculation includes a term $d E_{\gamma} / d \bar{v}=1.1 \mathrm{MeV}$, which is due to competing emission of prompt $\gamma$-rays [19]. This term is assumed to be constant for all mass splits. The deviation of the results indicates that the competition by prompt $\gamma$-rays has a dependence on the mass split.

\section{Conclusions}

Measurements of fission fragment properties and PFNs in resonance neutron induced fission have been presented. The presented results represent $25 \%$ of the expected total number of events. The fluctuation of fission fragment TKE's from resonance to resonance observed in Ref. [3] is confirmed. The fluctuations are largely explained by changes in the asymmetric mass distribution, increases in the TKE are correlated with an increase in mass yield in the heavy fragment mass range $\sim(125-135)$ u. No statistically significant fluctuation of $\bar{v}$ from resonance to resonance is observed at the present stage of the experiment.

Results on correlations of $\bar{v}$ with fission fragment properties show significant differences compared to earlier studies on this reaction, induced by thermal neutrons. The sawtooth shape of the average neutron multiplicity per fragment show more pronounced minima at $A \approx 130$ and $A \approx 80$. The TKE dependence of the neutron multiplicity per fission shows an inverse slope $\partial \mathrm{TKE} / \partial \bar{v}$ approximately $35 \%$ weaker than observed in 
earlier studies [23-26]. The difference can be explained by improved fission fragment TKE resolution in the present experiment. The average energy cost per emitted neutron is determined from the dependence of the neutron multiplicity on TKE for individual mass splits. The derived value $8.51 \mathrm{MeV} / \mathrm{n}$ is in reasonable agreement with the value of $8.6 \mathrm{MeV} / \mathrm{n}$ determined for ${ }^{252} \mathrm{Cf}$ by Nifenecker [19]. The present result should have strong impact on the modeling of both prompt neutron and prompt $\gamma$-ray emission in fission of the ${ }^{236} \mathrm{U}$ compound nucleus, specifically considering the strong differences observed in the TKE dependence of the prompt neutron multiplicity.

\section{References}

[1] C. Lubitz, R. Roussin, NEA/WPEC 18 (1999)

[2] C. De Saint Jean, R. McKnight, NEA/WPEC 34 (2014)

[3] F.-J. Hambsch et al., Nuclear Physics A 491, 56 (1989)

[4] F.-J. Hambsch et al., in Proc. Theory-1, EUR 24802 (Sinaia, Romania, 2011), p. 41

[5] R.E. Howe et al., Phys. Rev. C 13, 195 (1976)

[6] O. Litaize, O. Serot, Phys. Rev. C 82, 054616 (2010)

[7] O. Litaize, et al., The European Physical Journal A 51, 1 (2015)
[8] S. Lemaire et al., Physical Review C - Nuclear Physics 72 (2005), cited By 71

[9] N. Kornilov et al., Nuclear Physics A 789, 55 (2007)

[10] A. Göök et al., Nucl. Instr. Meth. A 830, 366 (2016)

[11] N. Kornilov, INDC(USA)-108 (2015)

[12] N. Kornilov et al., Nucl. Instr. Meth. A 599, 226 (2009)

[13] V. Verbinski et al., Nucl. Instr. Meth. 65, 8 (1968)

[14] M. Tajik et al., Nucl. Instr. Meth. A 704, 104 (2013)

[15] W. Mannhart, IAEA-TECDOC-410 (1986)

[16] A. Trkov et al., Nuclear Data Sheets 123, 8 (2015)

[17] F. Gönnenwein, in: The nuclear fission process (Ed. C. Wagemans, CRC Press, Boca Raton, Florida, 1991), chap. 8, pp. 287-473

[18] C. Wahl, Atomic Data and Nuclear Data Tables 39, 56 (1988)

[19] H. Nifenecker et al., 3rd IAEA Symp. on the Physics and Chemistry of Fission 2, 117 (1973)

[20] P. Geltenbort et al., Radiation Effects 93, 57 (1986)

[21] A. Göök et al., Phys. Rev. C 90, 064611 (2014)

[22] A. Gavron, Nucl. Inst. and Meth. 115, 99 (1974)

[23] K. Nishio et al., Nucl. Phys. A 632, 540 (1998)

[24] A. Vorobyev et al., G. Petrov, EPJ Web of Conf. 8, 03004 (2010)

[25] E.E. Maslin et al., Phys. Rev. 164, 1520 (1967)

[26] J.W. Boldeman et al., Aust. J. Phys. 24, 821 (1971)

[27] G. Audi et al., Chinese Physics C 36, 1287 (2012) 